\title{
AN APPROXIMATE SOLUTION FOR THE CONTACT AREA AND ELASTIC COMPLIANCE OF A SMOOTH PUNCH OF ARBITRARY SHAPE
}

\author{
J. R. BARber and D. A. Billings \\ Department of Mechanical Engineering and Applied Mechanics, University of Michigan, Ann Arbor, \\ MI 48109-2125, U.S.A.
}

(Received 13 March 1990; and in revised form 6 July 1990)

\begin{abstract}
An approximate solution is developed for the contact area and the load-penetiation relation for frictionless indentation of the elastic half-space by a punch of arbitrary profile. The method makes use of a previous result to the effect that the contact area in this problem is that which maximizes the total indenting force. An estimate of this force is obtained by applying the reciprocal theorem to the solution for indentation by a flat punch of the same plan-form, for which an approximate solution has recently been developed by Fabrikant. The method is illustrated using an example, the results of which are compared with a direct numerical solution using Hartnett's algorithm.
\end{abstract}

\section{INTRODUCTION}

The classical frictionless contact problem in which an elastic half-space is indented by a smooth rigid punch is nonlinear, since the extent of the contact area is not known a priori, but must be determined from inequality constraints stating that (i) the tractions in the contact area be non-tensile and (ii) the gap between the indenter and the deformed surface of the half-space be non-negative. The general problem may be solved by a variety of numerical (mostly variational or iterative) methods [1-5], but closed-form analytical solutions are known only for the classical Hertzian case, in which the indenting body has a quadratic profile and the contact area is elliptical.

If $P(A)$ is the force required to establish contact over some candidate contact area, $A$, it can be shown [6] that, for a fixed depth of indentation, the inequalities will be satisfied if and only if $A$ is chosen so as to maximize $P$. Furthermore, we can use Betti's reciprocal theorem to determine $P(A)$ for a punch of arbitrary profile $[7,8]$, provided we know the contact pressure distribution beneath a flat punch whose plan-form is $A$.

Of course, closed-form solutions of the flat punch problem are also only known for elliptical contact areas, so this result does not permit us to extend the catalogue of solutions to the general problem. However, Fabrikant [9] has recently developed an approximate solution to the flat punch problem, which shows quite good agreement with numerical data for quite a wide range of punch plan-forms.

In the present paper, we therefore use Fabrikant's solution [9] and the ideas of Barber [6] and Mossakovskii [7] to develop an approximate solution for the general frictionless contact problem. An example problem is solved and the results compared with a direct numerical solution using Hartnett's method [2].

\section{STATEMENT OF THE PROBLEM}

We consider the elastic half-space $z>0$ indented by a frictionless rigid punch, as shown in Fig. 1. The profile of the punch and its indentation are described through the function $f(x, y)$, which can be defined as the depth of the punch surface below the original undeformed position of the half-space surface, $z=0$.

We can now define the gap $g(x, y)$ between the punch and the half-space as

$$
g(x, y)=u_{z}(x, y, 0)-f(x, y),
$$

where $u_{z}(x, y, 0)$ is the normal elastic displacement of the half-space surface. 


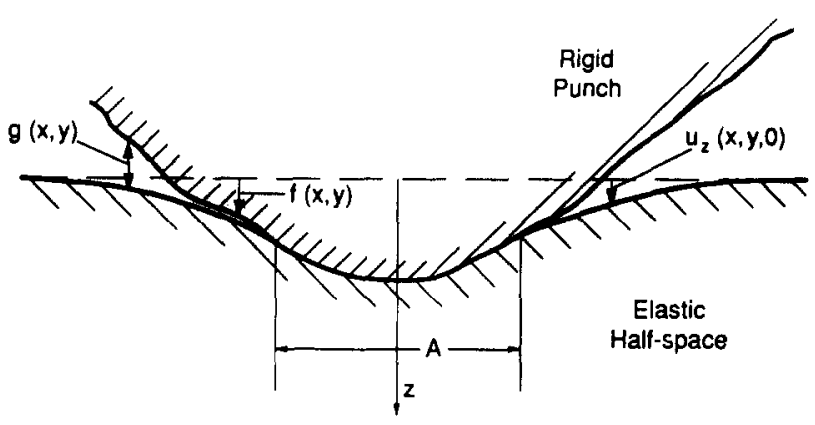

FIG. 1. The general indentation problem for a smooth rigid punch.

The gap must be zero within the contact region $A$ and must be positive in the separation region $\bar{A}$, since interpenetration of material is prohibited. We therefore have the two conditions

$$
\begin{aligned}
& u_{z}(x, y, 0)=f(x, y) ; \text { in } A \\
& u_{z}(x, y, 0)>f(x, y) ; \text { in } \bar{A} .
\end{aligned}
$$

To complete the definition of the frictionless contact problem, we require that the tangential traction be zero throughout the surface of the half-space, i.e.

$$
\sigma_{z x}=\sigma_{z y}=0 ; \text { all } x, y, z=0
$$

and that the contact pressure $p(x, y) \equiv-\sigma_{z z}(x, y, 0)$ be zero in the separation region $\bar{A}$ and positive (i.e. non-tensile) in the contact region $A$, i.e.

$$
\begin{aligned}
& p(x, y)=0 \text { in } \bar{A} \\
& p(x, y)>0 \text { in } A .
\end{aligned}
$$

If the contact region $A$ is specified, the equality conditions (2), (4) and (5) define a wellposed boundary value problem for the half-plane which has a unique solution. We can then define the total contact force

$$
P(A)=\iint_{A} p(x, y) \mathrm{d} x \mathrm{~d} y .
$$

The inequalities (3) and (6) serve to determine the extent of the contact area for the contact problem of Fig. 1, but it has been shown [6] that the value of $A$ which satisfies (3) and (6) is also that which maximizes the total force $P(A)$ given by equation (7).

\subsection{Determination of $\mathbf{P}(\mathbf{A})$}

The direct method of determining $P(A)$ would be to solve the boundary value problem defined by equations (2), (4) and (5) and then substitute the resulting contact pressure into (7). However, there is no general non-numerical method of solving the boundary value problem. We could of course use one of the numerical methods referred to in Section 1 above, in which case the problem would be reduced to a numerical optimization problem. However, the iteration involved in this optimization process would be just as time consuming as a direct iterative procedure based on the inequalities themselves, as used for example in the algorithm of Hartnett [2].

A more efficient approach, first introduced by Mossakovskii [7], is to use Betti's reciprocal theorem. We consider the simpler boundary value problem in which a frictionless rigid flat punch of plan-form $A$ is pressed into the half-space so that the indentation is a constant, $d^{*}$. This problem is of course defined by equations (2), (4) and (5) with $f(x, y)=d^{*}$. We denote the corresponding pressure distribution for this case by the function $p^{*}(x, y)$. 
We now apply the reciprocal theorem to this problem and the more general problem of equations (2), (4) and (5), obtaining the relation

$$
\iint_{A} p(x, y) d^{*} \mathrm{~d} x \mathrm{~d} y=\iint_{A} p^{*}(x, y) f(x, y) \mathrm{d} x \mathrm{~d} y .
$$

Since $d^{*}$ is by definition a constant, we can take it outside the integral in equation (8), obtaining

$$
P(A) \equiv \iint_{A} p(x, y) \mathrm{d} x \mathrm{~d} y=\frac{1}{d^{*}} \iint_{A} p^{*}(x, y) f(x, y) \mathrm{d} x \mathrm{~d} y .
$$

Thus, we can obtain an expression for the total force $P(A)$ in the general contact problem, provided we can determine the contact pressure $p^{*}(x, y)$ for a flat punch of plan-form $A$.

\subsection{Rayleigh-Ritz approximation}

Closed-form solutions of the flat punch problem are known only for the case where the contact area $A$ is circular, elliptical or an infinite strip. Thus, the above procedure does not provide a general non-numerical method for determining the contact area in the problem of Fig. 1. However, it does permit us to develop approximate solutions in the Rayleigh-Ritz sense-i.e. to determine the "best" elliptical approximation to the actual contact area-and this procedure will also yield a corresponding approximation to the load-penetration relation. This is potentially very useful in situations where the contact condition is only important in the sense of defining a nonlinear spring characteristic for input to a larger static or dynamic structural analysis problem.

\subsection{Use of Fabrikant's solution}

A more speculative extension of the method can be made, using a recent approximate solution for the flat punch problem due to Fabrikant [9]. Briefly, Fabrikant argues that the contact pressure under an arbitrary flat punch will be given approximately by

$$
p(r, \theta)=\frac{4 \mu d^{*} a(\theta)}{(1-v) L \sqrt{a^{2}(\theta)-r^{2}}}
$$

where $a(\theta)$ defines the boundary of the contact region $A$ in polar coordinates, $\mu, v$ are respectively the modulus of rigidity and Poisson's ratio for the half-space material and

$$
L=\int_{0}^{2 \pi} a(\theta) \mathrm{d} \theta .
$$

The origin for the system of polar coordinates should be taken at the centroid of the area $A$ [9].

The traction distribution (10) is exact for the three "closed-form" cases where $A$ is a circle, an ellipse or a strip and Fabrikant demonstrates that it compares favorably with previous approximate and numerical solutions for a wide variety of contact area shapes, particularly in respect of the predicted relation between load and penetration.

We now substitute this expression into equation (9), obtaining an approximate expression $^{\dagger}$ for the force $P(A)$ in the form

$$
P(A)=\frac{4 \mu}{(1-v) L} \int_{0}^{2 \pi} \int_{0}^{a(\theta)} \frac{a(\theta) f(r, \theta) r \mathrm{~d} r \mathrm{~d} \theta}{\sqrt{a^{2}(\theta)-r^{2}}} .
$$

Note that this expression can be generalized to the case of two deformable bodies by replacing $\mu /(1-v)$ by $\left[\left(1-v_{1}\right) / \mu_{1}+\left(1-v_{2}\right) / \mu_{2}\right]^{-1}$ and $f(r, \theta)$ by the interpenetration of the two undeformed bodies.

The problem is now to choose the function $a(\theta)$ so as to maximize $P(A)$.

\footnotetext{
+Notice that the expression is approximate only insofar as Fabrikant's solution for the flat punch problem is approximate.
} 


\section{SOLUTION OF THE OPTIMIZATION PROBLEM}

Suppose we first examine a sub-class of variations of $a(\theta)$ for which $L$ [see equations $(10,11)]$ is constrained to be a prescribed constant. A particular variation which satisfies this constraint is

$$
\begin{aligned}
\Delta a(\theta) & =\delta ; \theta_{1}-\varepsilon<\theta<\theta_{1}+\varepsilon \\
& =-\delta ; \theta_{2}-\varepsilon<\theta<\theta_{2}+\varepsilon \\
& =0 ; \text { all other } \theta
\end{aligned}
$$

where $\delta \ll a, \varepsilon \ll 2 \pi$, the corresponding variation in $P$ being

$$
\begin{aligned}
\Delta P= & \frac{8 \mu \delta}{(1-v) L}\left[\frac{\mathrm{d}}{\mathrm{d} a\left(\theta_{1}\right)} \int_{0}^{a\left(\theta_{1}\right)} \frac{a\left(\theta_{1}\right) f\left(r, \theta_{1}\right) r \mathrm{~d} r}{\sqrt{a^{2}\left(\theta_{1}\right)-r^{2}}}\right. \\
& \left.-\frac{\mathrm{d}}{\mathrm{d} a\left(\theta_{2}\right)} \int_{0}^{a\left(\theta_{2}\right)} \frac{a\left(\theta_{2}\right) f\left(r, \theta_{2}\right) r \mathrm{~d} r}{\sqrt{a^{2}\left(\theta_{2}\right)-r^{2}}}\right]
\end{aligned}
$$

for sufficiently small $\delta, \varepsilon$.

For $P(A)$ to be a maximum in the sub-class of constant $L, \Delta P$ must be zero and hence the two integrals in (14) must be equal and opposite. But $\theta_{1}$ and $\theta_{2}$ can be chosen at random and hence we must have

$$
\left(\frac{\partial}{\partial a} \int_{0}^{a(\theta)} \frac{a(\theta) f(r, \theta) r \mathrm{~d} r}{\sqrt{a^{2}(\theta)-r^{2}}}\right)_{\theta}=D
$$

for all $\theta$ where $D$ is an as yet unknown constant (i.e. $D$ is independent of $\theta$ ).

If $D$ were known, (15) would constitute an equation sufficient to determine ${ }^{\dagger}$ the function $a(\theta)$. We can therefore solve the original problem by treating $D$ as an unknown, solving for $a(\theta)$ and hence $P_{\max }(D)$, which is now a function of $D$ only, and finally choosing $D$ to maximize $P_{\max }(D)$.

Notice incidentally that $L$ drops out of the constrained optimization problem, during which it is a constant. There is of course a parametric relation between $L$ and $D$, so that the final stage of the process also determines the value of $L$.

\section{EXAMPLE}

To illustrate the procedure, we consider the case in which the symmetric vertex of a tetrahedral punch is pressed into the half-space, the inclination of the tetrahedral faces to the half-space surface in the undeformed state being denoted by $\alpha$. The interpenetration function $f(r, \theta)$ for this case is

$$
\begin{aligned}
f(r, \theta) & =d_{0}-r \tan \alpha \cos \theta ; \quad-\frac{\pi}{3}<\theta<\frac{\pi}{3} \\
& =d_{0}-r \tan \alpha \cos \left(\theta-\frac{2 \pi}{3}\right) ; \quad \frac{\pi}{3}<\theta<\pi \\
& =d_{0}-r \tan \alpha \cos \left(\theta+\frac{2 \pi}{3}\right) ; \quad-\pi<\theta<-\frac{\pi}{3}
\end{aligned}
$$

where $d_{0}$ is the indentation of the vertex.

\footnotetext{
${ }^{\dagger}$ The expression for $a(\theta)$ may turn out to be multivalued, but it is a simple matter to examine the corresponding solutions and pick that which gives the largest $P(A)$.
} 


\subsection{The approximate solution}

Substituting equation (16) into equation (15) and performing the integration and differentiation, we obtain

$$
2 d_{0} a-\frac{3 \pi a^{2} \tan \alpha \cos \theta}{4}=D, \quad-\frac{\pi}{3}<\theta<\frac{\pi}{3}
$$

which has the solution

$$
a(\theta)=\frac{4 d_{0} \pm 2 \sqrt{4 d_{0}^{2}-3 \pi D \tan \alpha \cos \theta}}{3 \pi \tan \alpha \cos \theta} ; \quad-\frac{\pi}{3}<\theta<\frac{\pi}{3} .
$$

This result is repeated in the two remaining ranges of equation (16), with $\theta$ replaced by $(\theta-2 \pi / 3),(\theta+2 \pi / 3)$, respectively.

Notice that this equation has a physically admissible solution for all $\theta$ if and only if $D<4 d_{0}^{2} / 3 \pi \tan \alpha$.

The force, $P_{\max }(D)$, is now obtained by substituting (18) into (12), using (11) to evaluate $L$ and performing the integrations. It turns out that $P_{\max }(D)$ increases monotonically with $D$ in the admissible range and hence that the value of $D$ which maximizes $P(A)$ is $4 d_{0}^{2} / 3 \pi \tan \alpha$.

With this value, the expression for $a(\theta)$ simplifies to

$$
a(\theta)=\frac{4 d_{0}(1 \pm \sqrt{1-\cos \theta})}{3 \pi \tan \alpha \cos \theta} ; \quad-\frac{\pi}{3}<\theta<\frac{\pi}{3}
$$

etc.

The corresponding indentation force is obtained by substituting this result into equations (11) and (12) and performing the integrations. The larger value corresponds to the choice of the positive sign in equation (19) and is

$$
P=\frac{1.6960 \mu d_{0}^{2}}{(1-v) \tan \alpha}
$$

\subsection{A Rayleigh-Ritz approximation}

It is interesting to compare this result with that obtained using the Rayleigh-Ritz procedure of Section 2.2 above. Since the tetrahedral punch has symmetry about three planes, the "best ellipse" is a circle centred on the vertex of unknown (but now constant) radius, $a$. The corresponding force, $P(A)$, is then found to be

$$
\begin{aligned}
P(A) & =\frac{6 \mu}{\pi(1-v)} \int_{-\pi / 3}^{\pi / 3} \int_{0}^{a} \frac{\left(d_{0}-r \tan \alpha \cos \theta\right) r \mathrm{~d} r \mathrm{~d} \theta}{\sqrt{a^{2}-r^{2}}} \\
& =\frac{4 \mu}{(1-v)}\left[a d_{0}-\frac{3 \sqrt{3} a^{2} \tan \alpha}{8}\right]
\end{aligned}
$$

from equations (12) and (16).

We determine the contact radius, $a$, by applying the condition $\mathrm{d} P / \mathrm{d} a=0$, with the result

$$
a=\frac{4 d_{0}}{3 \sqrt{3} \tan \alpha} ; \quad P=\frac{1.5396 \mu d_{0}^{2}}{(1-v) \tan \alpha} .
$$

\subsection{Comparison with a numerical solution}

The accuracy of these approximations can conveniently be tested by solving the same problem using a direct numerical method, a convenient algorithm being that of Hartnett [2], in which the contact pressure is represented as piecewise constant over a system of rectangular cells on the surface of the half-space. Any desired accuracy can be obtained by increasing the number of cells.

Figure 2 shows the contact region for the tetrahedral punch predicted by Hartnett's method using a mesh of $49 \times 49$ cells. Superimposed on the same figure, we show the contact area shape predicted using Fabrikant's approximation [equation (19)] and a circle of radius $4 d_{0} / 3 \sqrt{3} \tan \alpha$ [equation (23)]. Clearly the Rayleigh-Ritz approach gives a good 


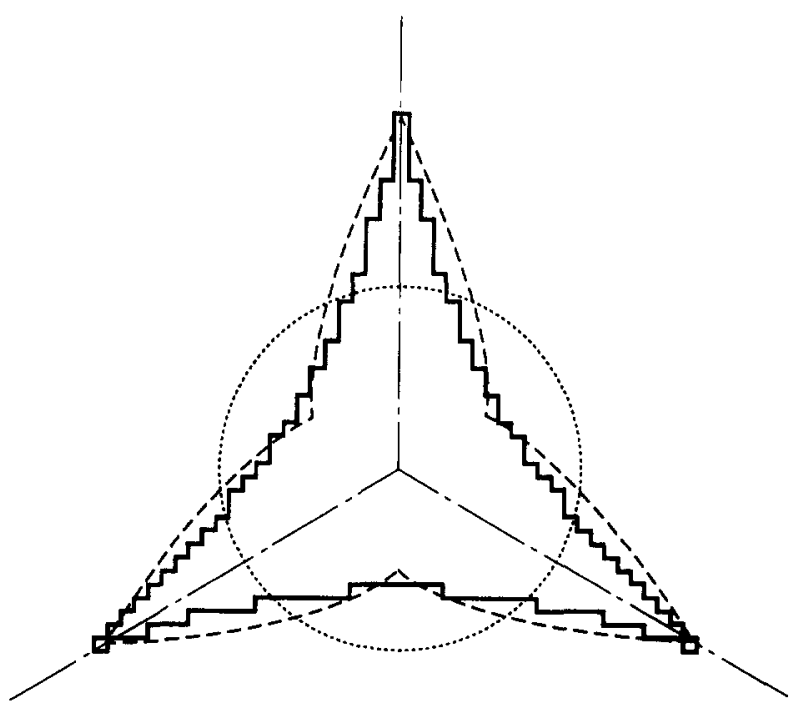

Fig. 2. Contact region for the tetrahedral punch:---- numerical solution equation (23), --- equation (19).

TABLE 1.

\begin{tabular}{ccccccccccc}
\hline$n$ & 3 & 4 & 5 & 6 & 7 & 8 & 10 & 15 & 20 & 50 \\
\hline$\frac{3 \pi D \tan \alpha}{4 d_{0}^{2}}$ & 1.000 & 0.853 & 0.809 & 0.789 & 0.778 & 0.770 & 0.763 & 0.757 & 0.754 & 0.750 \\
\hline$\frac{(1-v) P \tan \alpha}{\mu d_{0}^{2}}$ & 1.696 & 1.449 & 1.373 & 1.339 & 1.320 & 1.308 & 1.295 & 1.283 & 1.279 & 1.274 \\
\hline
\end{tabular}

estimate of the overall size of the contact area, but does not adequately describe its shape, whereas the solution using Fabrikant's approximation is quite good as to both size and shape.

The numerical solution predicts an indentation force of

$$
P=\frac{1.7725 \mu d_{0}^{2}}{(1-v) \tan \alpha}
$$

which compares favorably with both methods, indicating an error of $13 \%$ using the Rayleigh-Ritz method and only $4.3 \%$ using Fabrikant's approximation.

We therefore conclude that the methods of this paper provide a useful and reasonably accurate way of estimating the force displacement relation for non-Hertzian smooth elastic contacts.

\subsection{The general polyhedral punch}

The above example can be regarded as a special case of the $n$-faced polyhedral punch for which the interpenetration function is

$$
f(r, \theta)=d_{0}-r \tan \alpha \cos \theta ; \quad-\frac{\pi}{n}<\theta<\frac{\pi}{n}
$$

repeated in the remaining $(n-1)$ intervals.

Equations (17) and (18) still hold for this more general problem in $-\pi / n<\theta<\pi / n$, but the corresponding value of $P_{\max }(D)$ from equation (12) has a maximum in the admissible range (i.e. not at the end point $D=4 d_{0}^{2} / 3 \pi \tan \alpha$ ) for $n>3$. 
Values of $D$ and the total force $P$ for various $n$ are given in Table 1. The predicted contact area can then be recovered from (18) in $-\pi / n<\theta<\pi / n$ and is repeated in the remaining intervals.

As $n \rightarrow \infty, D \rightarrow d_{0}^{2} / \pi \tan \alpha$ and $P \rightarrow 4 \mu d_{0}^{2} / \pi(1-v) \tan \alpha$, agreeing with the classical solution for indentation by a conical punch due to Love [10].

Acknowledgements-J. R. Barber is pleased to acknowledge the support of the National Science Foundation under Contract No. MSM-8719073.

\section{REFERENCES}

1. B. PAul and J. HaShemi, Contact pressure on closely conforming elastic bodies. J. appl. Mech. 48, 543 (1981).

2. M. J. Hartnett, A general numerical solution for elastic body contact problems. ASME Symp. Solid Contact and Lubrication, Chicago, pp. 51-66 (1980).

3. R. H. BeNtAll and K. L. Johnson, Slip in the rolling contact of two dissimilar elastic rollers. Int. J. Mech. Sci. 9, 389 (1967).

4. J. J. KalKer and Y. VAN RANDEN, A minimum principle for frictionless elastic contact with applications to non-Hertzian half-space contact problems. J. Engng Math. 6, 193 (1973).

5. N. KIKUChI and J. T. Oden, Contact Problems in Elasticity: A Study of Variational Inequalities and Finite Element Methods. SIAM, Philadelphia (1988).

6. J. R. BARBER, Determining the contact area in elastic indentation problems. J. Strain Anal. 9, 230 (1974).

7. V. I. MossakovsKII, Application of the reciprocity theorem to the determination of the resultant forces and moments in three-dimensional contact problems (in Russian). PMM 17, 477 (1953).

8. R. T. Shield, Load-displacement relations for elastic bodies. Z. angew. Math. Phyz. 18, 682 (1967).

9. V. I. FABRIKANT, Flat punch of arbitrary shape on an elastic half-space. Int. J. Engng Sci. 24, 1731 (1986).

10. A. E. H. Love, Boussinesq's problem for a rigid cone. Q. J. Math. 10, 161 (1939). 